\title{
1.4-Arylation of $\beta$-(4-acetylaminobenzoyl)acrylic Acid with Activated Aromatic Hydrocarbons under Friedel-Crafts Conditions and Some Studies with the Products
}

\author{
S.A. Rizk", M.A. ELHashash and M.M. Aburzeza \\ Chemistry Department, Faculty of Science, Ain Shams \\ University Cairo, Egypt.
}

\begin{abstract}
HE BEHAVIUOR of 3-(4-acetylaminobenzoyl) prop-2-enoic acid 1 with $m$-xylene andlor $p$-xylene under Friedel-Craft's reaction conditions yielded 2- ( 2,4-Dimethyl and/or 2,5-dimethyl ) phenyl-3-(4-acetylaminobenzoyl)propanoic acids $(2 \mathrm{a}, \mathrm{b})$ and thiaMicheal of acid 1 afforded 2- phenyl sulfanyl-3-(4-acetylamino benzoyl propanoic acid (3). Interaction of acids 2,3 with $\mathrm{N}_{2} \mathrm{H}_{4}$, $\mathrm{AC} 2 \mathrm{O}, \mathrm{NH} 2 \mathrm{OH} . \mathrm{HCl}$ and $\mathrm{PhNHNH} 2$, yielded pyridazinone 4 , Furanone 51 , 2oxazine 6 and 2-phenyl pyridazinone derivatives, respectively. Treatment of pyridazinone 4 with different interesting alkyl halides afforded the pyridazine derivatives 8 .
\end{abstract}

Keywords: 3-Aroyl prop-2-enoic acid. Pyridazinone. Furanone, oxazinone and Alkyl pyridazine .

Pyridazinone derivatives were reported to exhibit diverse pharmacological activities anti-depressant ${ }^{(1)}$, antihypertensive ${ }^{(2,3)}$, anti-thrombotic ${ }^{(4)}$, anticonvulsant ${ }^{(5)}$, cardiotonic ${ }^{(6)}$, antibacterial ${ }^{(7)}$, diuretics ${ }^{(8)}$, anti HIV ${ }^{(9)}$, as anticancer ${ }^{(10 a)}$ and as analgesic agent ${ }^{(10 b)}$. Some pyridazinone derivatives like indolidan ${ }^{(11)}$ bemoradan ${ }^{(12)}$, pyimobendan ${ }^{(13)}$, levosimedan ${ }^{(14)}$, menaprine ${ }^{(15)}$, emorfazone $^{(16)}$ and azanrinone ${ }^{(17)}$, already appeared in the clinical market. It is observed that the 6-phenyl-2(3H)pyridazinone residue considered as pharmacophoric group in the position six in pyridazinone becomes more active than the pyridazenone moiety. Also, from the medicinal chemistry research point of view the presesce of aryl and alkyl groups as the position of 2- and 6- in pyridazinone made ten times more active than itself. In recently published papers ${ }^{(18-19)}$ the pyridazinone carrying the aryl and alkyl group in position 2- and 6- are more potent . So we have synthesized some new pyridazinone derivatives carring the lipophilic aryl and alkyl groups in the positions 2-, 4- and 6- . The FriedelCrafts acylation of aromatic hydrocarbon with maleic anhydride afforded $\beta$ aroyl propenoic acid. Interaction of $\beta$-aroyl prop-2-enoic acid with aromatic hydrocarbon in the presesce of a Lewis acid anhydrous $\mathrm{AlCl}_{3}$ under FriedelCrafts Condition afforded $\alpha$-aryl- $\beta$-aroyl propanoic acid. Treatment of the product with $\mathrm{N}_{2} \mathrm{H}_{4}$ and for $\mathrm{PhNHNH}_{2}$ afforded the desired target .

*Samehrizk2006@gmail.com 


\section{Results and Discussion}

The $\beta$ - aroylacrylic acid derivative , 4 (4-acetylamino phenyl)-4-oxo-but-2enoic acid $(1)^{(20-29)}$ has interacted with hydrocarbons $m$-xylene and $p$-xylene in the presence of the anhydrous aluminium chloride under Friedel Crafts reaction to afford 2-(2,4 dimethyl and lor 2,5dimethyl) phenyl-3(4-acetylamino benzoyl) propionic acid (2). The acids 2 used as key starting materials for synthesizing the interesting heterocyclic systems, the structure of compounds 2 was established by their correct analytical data and their IR spectra which exhibited strong absorption at the regions 1688-1670 and 3330$3160 \mathrm{~cm}^{-1}$ attributable to $v \mathrm{CO}$ and $v \mathrm{NH}$, respectively. EIMS for compounds 2 exhibited $\mathrm{m} / \mathrm{z} 339\left(\mathrm{M}^{+}\right)$.The reaction takes place via nucluphilic addition of hydrocarbon to the $\alpha, \beta$ unsaturated carbonyl moiety of the acid 1 that gave the less electrostatic repulsion transition state as below



On the other hand, interaction of acid $1^{(30,31)}$ with thiophenol in benzene in the presence of piperidine as acatalyst, it afforded thia-Michael adduct of 3(4-acetylaminobenzoyl) -2-phenyl mercapto propionic acid 3, its IR spectrum exhibits strong absorption hands at $1689-1670,3300-3170 \mathrm{~cm}^{-1}$ attributable to $v \mathrm{CO}$ and $v \mathrm{NH}$, respectively. The ${ }^{1} \mathrm{H}-\mathrm{NMR}$ spectrum of compound 3 revealed singlet at $\delta 2.15 \mathrm{ppm}$ assigned to $\mathrm{CH}_{3}$ group, two douplet at $\delta 2.3 \mathrm{ppm}$ assigned to diasteriotopic protons $\left(\mathrm{CH}_{2}-\mathrm{CH}\right), 3.7 \mathrm{ppm}$ assigned to methine proton, multiplet at 6.8-7.8 assigned for aromatic protons and finally two exchangeable singlet at $\delta 8.1$ and $13.1 \mathrm{ppm}$ consistent with protons of ( $\mathrm{NH}$ and $\mathrm{OH})$, respectively. EIMS for compound 3 exhibited $\mathrm{m} / \mathrm{z} 343$ corresponding to $\left(\mathrm{M}^{+}\right)$. Pyridazinone is an important clam of heterocycles, which have been the subject of extreme research particularly are the pharmaceutical and agrochemical which have their broad activities such as antihypertensive activity and anti inflammatory ${ }^{(32-34)}$, their synthesis application have been compressively reviewed ${ }^{(35,36)}$, anticipated NSAID ${ }^{(37,38)}$. Thus, when acids 2,3 were reacted with $\mathrm{N}_{2} \mathrm{H}_{4}$ in boiling ethanol. They afforded 6 (4- acetylamino phenyl)-4-(2,4 and /or 2,5 dimethyl phenyl and phenyl mercapto)2,3,4,5 tetrahydro $3(2 \mathrm{H})$ pyridazinone 4 (Scheme 1). IR spectra for compounds 4 exhibit strong absorption bands at $1690-1670 \mathrm{~cm}^{-1}$ and $3288-3285 \mathrm{~cm}^{-1}$ corrsponding to $v \mathrm{CO}$ and $v \mathrm{NH}$, respectively. The ${ }^{1} \mathrm{H}-\mathrm{NMR}$ spectrum of compound $4_{\mathrm{b}}$ revealed singlet at $\delta 2.2 \mathrm{ppm}$ assigned to 3 methyl groups, $2.8 \mathrm{ppm}$ assigned 2.8 (2dd, $2 \mathrm{H}$,diasterotopic protons), 3.4 (dd, $1 \mathrm{H}, \mathrm{CH}-\mathrm{CO}$, pyridazine moiety), multiplet at 6.8$7.4 \mathrm{ppm}$ assigned to aromatic protons and exchangeable protons, singlet at 8.5 and $13 \mathrm{ppm}$ corresponding to $\mathrm{NH}$ and $\mathrm{OH}$ groups, respectively. EIMS for compound $4 \mathrm{c}$ exhibited $\mathrm{mlz} 339$ corresponding to $\left(\mathrm{M}^{+}\right)$. 


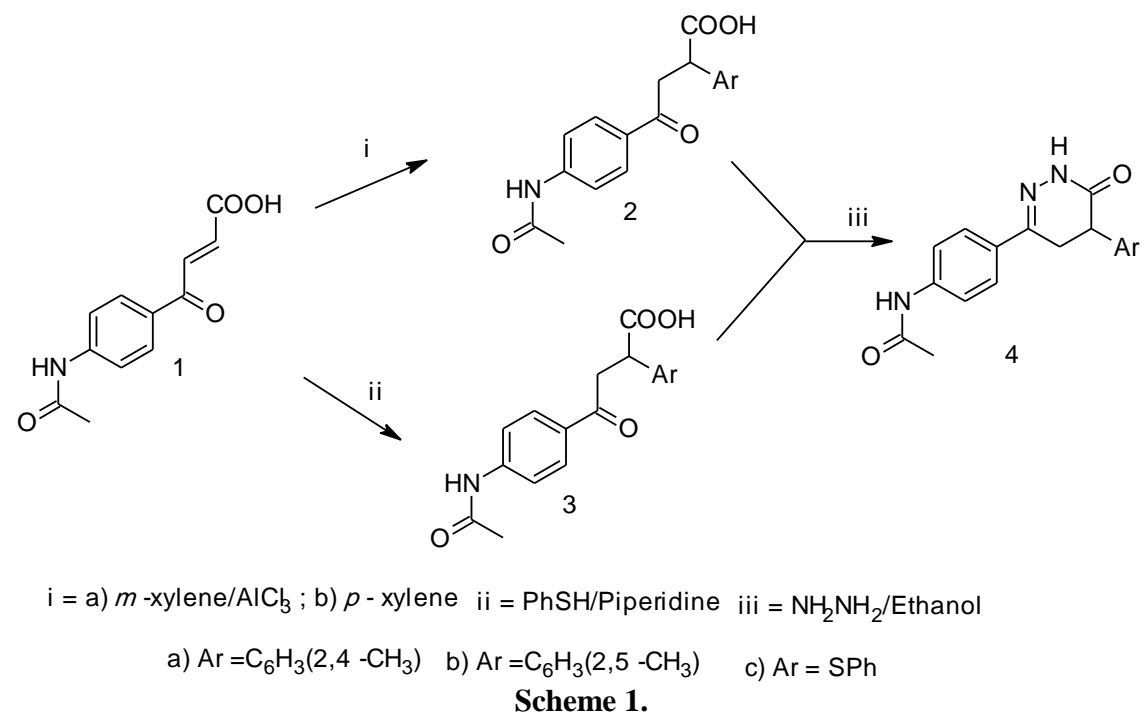

Furthermore due to their common occurrence in nature, oxygen containing in $2(5 \mathrm{H})$ - Furanone has UV-induced unimolecular photochemistry ${ }^{(39)}$. Its important target for synthesis either as final product or as useful synthetic intermediates, the synthesis of lactones can be achieved by the lactonization of hydroxyl acids Baeyer- villiger oxidation,the insertion of a carbonyl group by transition metals, intramolecular cyclization of diones ${ }^{(40)}$.Thus, when acids 2,3 were allowed to react with acetic anhydride under reflux and for heating or in water bath for $1 \mathrm{hr}$, afforded 5-(4-acetamido phenyl)-3-(2,4-dimethyl/2,5 dimethyl and /or phenyl mercapto)- $2(3 \mathrm{H})$ furanone 5. IR spectra revealed strong absorption bands at $1762-1750 \mathrm{~cm}^{-1}$ attributable to vCO. The ${ }^{1} \mathrm{H}-$ NMR spectrum of compound $5_{\mathrm{b}}$ in DMSO exhibited signals at $\delta 2.5 \mathrm{ppm}$ assigned to 3-methyl groups, $4 \mathrm{ppm}$ assigned to chiral center, doublet at 6.7 $\mathrm{ppm}$ assigned to olefinic protons in furanone ring, 7.5-7.9 $\mathrm{ppm}$ multiplet aromatic protons and exchangeable proton of $(\mathrm{NH})$ groups at $13.5 \mathrm{ppm}$. EIMS for compounds $5,5_{\mathrm{c}}$ exhibited $\mathrm{mlz} 322$ and 325 corresponding to $\left(\mathrm{M}^{+}\right)$, respectively. On the other hand, when compounds 2,3 were allowed to react with hydroxyl amine hydrochloride in boiling pyridine afforded 3-(4acetomido phenyl)-5- (2,4 or 2,5 dimethyl phenyl and /or phenyl mercapto)-1,2 oxazin-6-one(6).IR spectra revealed strong absorption bands at 1735 and 3140$3147 \mathrm{~cm}^{-1}$ attributable to $v \mathrm{CO}, \mathrm{vNH}$, respectively. Several studies have indicated that $\mathrm{NH}$ group to $\mathrm{CO}$ group and azine system may be an essential structural requirement in the binding of $3(2 \mathrm{H})$-pyridazinone to variety of biological receptors ${ }^{(41)}$. However , the numerous syntheses of $3(2 \mathrm{H})$ pyridazinones that have been published in recent years have made only limited progress in terms of the efficient protection of the 2-position in the heterocyclic ring. Although all structural studies on this nucleus have shown that $3(2 \mathrm{H})$ pyridazinones exist in the keto from ${ }^{(42)}$. Reaction involving ambident ring that possess a tautomeric structure are often inefficient and lack regio 
control. Thus treatment of compound (3) with phenyl hydrazine in boiling ethanol afforded 2-phenyl-4-(phenyl mercapto)-6-(4-acetyl aminophenyl)3(H) pyridazinone (7) which was established by its correct analytical data. IR spectrum exhibits strong absorption bands at $1687-1650 \mathrm{~cm}^{-1}$ corresponding to two carbonyl groups $v \mathrm{CO}$ and $3279 \mathrm{~cm}^{-1}$ for $v \mathrm{NH}$ (Scheme 2).

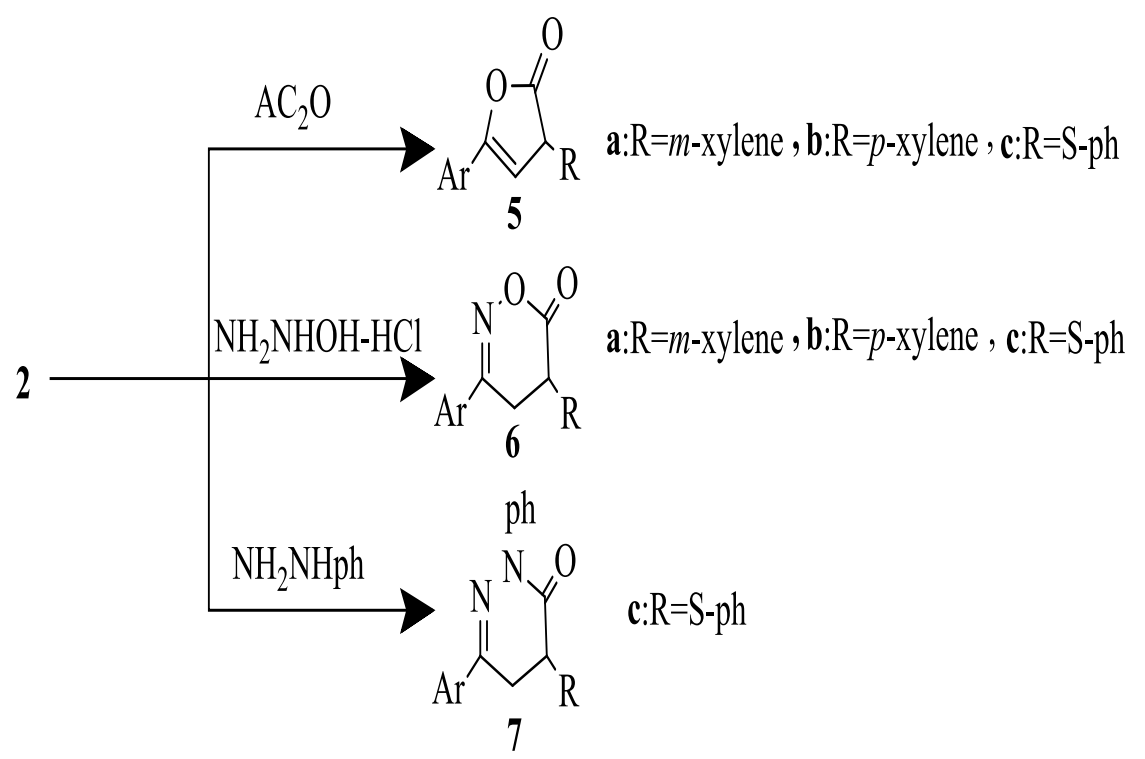

Scheme 2 .

Moreover, the authors used the pyridazinone as starting material to afford 2alkyl pyridazinone derivatives in which the position-2 is blocked in lactam structure . So, interaction of $3(2 \mathrm{H})$ pyridazinone derivatives $4_{a}$ and $4 \mathrm{~b}$ with different alkyl halides in pyridine, afforded the corresponding 2-alkyl pyridazinone derivatives 8 . The structure of compounds 8 was estabalished by their correct analytical data and IR spectra which exhibited strong absorption bands at the regions $1730-1670 \mathrm{~cm}^{-1}$ attributable to $v \mathrm{CO}$ for all derivatives 8 , the compounds $8_{\mathrm{k}}$ and $8_{1}$ have $v \mathrm{CO}$ at 1670 broad bands due to vibrational coupling of 2 carbonyl groups attached which by good inductor atom, e.g nitrogen atom (-CO-N-CO-) and the derivatives $8_{\mathrm{i}}$ and $8_{\mathrm{j}}$ have 2 carbonyl regions at 1730 and $1745 \mathrm{~cm}^{-1}$ due to ester group. The ${ }^{1} \mathrm{H}-\mathrm{NMR}$ of $8_{1}$ in DMSO $1.1\left(\mathrm{t}, 3 \mathrm{H}, \mathrm{CH}_{2} \mathrm{CH}_{3}\right) 2.3 \quad\left(\mathrm{~s}, 9 \mathrm{H}, \mathrm{CH}_{3}\right)[3.1(2 \mathrm{dd}, 2 \mathrm{H}$, disterotopicprotons), $3.7\left(\mathrm{dd}, 1 \mathrm{H}, \mathrm{CH}\right.$ of methine in pyridazine moiety],4.1(q, $\left.2 \mathrm{H}, \mathrm{CH}_{2} \mathrm{CH}_{3}\right), 4.8(\mathrm{~s}, 2 \mathrm{H}, \mathrm{N}-$ $\left.\mathrm{CH}_{2}\right)$ 7.17.9(m,7H,ArH) . EIMS for compound $8_{\mathrm{b}}$ and $8_{\mathrm{g}}$ exhibited $\mathrm{m} / \mathrm{z} 348$ and 426 , respectively corresponding to $\mathrm{M}^{+}$. The compound $8_{\mathrm{k}}$ exhibited mlz 336 corresponding to $\left(\mathrm{M}^{+}-\mathrm{COCH}_{3}\right)$. 
<smiles>CC(=O)Nc1ccc(C2=NNC(=O)C(Br)C2)cc1</smiles><smiles>CC(=O)Nc1ccc(C2=NNC(=O)C(Br)C2)cc1</smiles>

$\mathrm{Ar}=\mathrm{C}_{6} \mathrm{H}_{3}\left(2,4-\mathrm{CH}_{3}\right)$

$\mathrm{Ar}=\mathrm{C}_{6} \mathrm{H}_{3}\left(2,5-\mathrm{CH}_{3}\right)$
a) $\mathrm{CH}_{3} \quad$ c) $\mathrm{C}_{2} \mathrm{H}_{5}$
e) $\mathrm{CH}_{2}=\mathrm{CH} \mathrm{CH}_{2}$
g) $\mathrm{Ph} \mathrm{CH}_{2}$ i) $\mathrm{CH}_{3} \mathrm{CO}$
k) $\mathrm{CH}_{2} \mathrm{COOEt}$

b) $\mathrm{CH}_{3}$ d) $\mathrm{C}_{2} \mathrm{H}_{5}$

f) $\mathrm{CH}_{2}=\mathrm{CH} \mathrm{CH}$

h) $\mathrm{Ph} \mathrm{CH}_{2}$ j) $\mathrm{CH}_{3} \mathrm{CO}$

l) $\mathrm{CH}_{2} \mathrm{COOEt}$

Scheme 3.

\section{Experimental}

All melting points are uncorrected. Elemental analyses were carried out in the Microanalytical Center, Cairo University, Egypt. IR spectra were recorded in (KBr) disks on Shimadzu FTIR 8101Pe and ${ }^{1} \mathrm{H}-\mathrm{NMR}$ spectra recorded on a Varian $300 \mathrm{MHz}$ in $\left(\mathrm{CDCl}_{3}\right)$ or $\left(\mathrm{DMSO}_{-} \mathrm{d}_{6}\right)$ as solvents, (chemical recorded on Shimadzu GCMS-QP-1000EX mass spectrometer at $70 \mathrm{eV}$. Homogeneity of all compounds synthesized was checked by TLC. Characterization data of the various prepared compounds are given in Table 1.

\section{2-(3,4-Dimethyl and / or2,4dimethyl) phenyl-3-(4-acetylamino) benzoylpropionic acids $(2 a, b)$}

A solution of the 3(4-acetylamino) benzoyl acrylic acid $1(2.4 \mathrm{~g}, 0.01 \mathrm{~mol})$ in $m$-xylene and/or $p$-xylene $(50 \mathrm{ml})$ was treated with anhydrous aluminum chloride $(0.04 \mathrm{~mol})$ and the mixture was heated on the water bath for $10 \mathrm{hr}$. The mixture was treated with icelHCl. The organic layer was washed with water, and the excess solvent was removed by steam distillation. The organic material was extracted by ether. The ethereal layer was washed by $10 \%$ aq $\mathrm{Na}_{2} \mathrm{CO}_{3}$ solution, and was acidified by dil $\mathrm{HCl}$. The solid was separated out , filtered off , dried and recrystallized from the proper solvent to afford $2 a$ and $b$. 
IR Spectra for compounds $2 \mathrm{a}$ and $2 \mathrm{~b}$ exhibit vOH (b) 3330 , vCHAr 3050, v CHAli 2950,vCO1688-1670 $\mathrm{cm}^{-1}{ }^{1} \mathrm{H}-\mathrm{NMRspectrum}$ for 2a in DMSO 2.45 (s,9H,CH3), 3.1 (2dd,2H,diasterotopic protons), 3.9(dd,1H, CH-COO),6.87.8(m,7H,ArH), $11.2(\mathrm{~s}, 1 \mathrm{H}, \mathrm{COO}), 13.2(\mathrm{~s}, 1 \mathrm{H}, \mathrm{NH})$.EIMS appear $\mathrm{m} / \mathrm{z}$ at 339 corresponding to molecular ion peak.

2-Phenyl sulfanyl-4-oxo-(4-acetylamino) phenyl-propanoic acid (3)

A mixture of 3-(4-acetylaminobenzoyl)-prop-2-enoic acid $(2.4 \mathrm{~g}, 0.01 \mathrm{~mol})$ and Thiophenol 0.01 mole $(1 \mathrm{ml})$ in $20 \mathrm{ml}$ dry benzene and drops of piperidine for $4 \mathrm{hr}$. The product that separated was recrystallized from ethanol to give compound 3. IR Spectrum for compound 3 exhibited vNH 3300,vCHAr 3050, vCO $1689-1670 \mathrm{~cm}^{-1}{ }^{1} \mathrm{H}-\mathrm{NMR}$ spectrum for 3 in DMSO $2.15(\mathrm{~s}, 3 \mathrm{H}), 2.3$ (2dd,2H,diasterotopic protons), 3.7( $\mathrm{dd}, 1 \mathrm{H}, \mathrm{CH}-\mathrm{COOH}), 6.8-7.8 \quad(\mathrm{~m}, 9 \mathrm{H}, \mathrm{ArH})$ ), 8.1(s,1H,COOH), 13.1(s,1H,NH).

4-(2,4-or2,5 Dimethyl)phenyl and/or 4-phenyl sulfanyl 6-(4-acety aminophenyl)1, 4, 5,6-tetrahydro-3(2H)-pyridazinone (4)

A mixture of propionic acid derivatives $2,3 \quad(0.01 \mathrm{~mol})$ and hydrazine hydrate $(0.5 \mathrm{~mL}, 0.01 \mathrm{~mol})$ was heated under reflux in butanol $(30 \mathrm{ml})$ for $3 \mathrm{hr}$. The reaction mixture was concentrated.The solid was separated out, filtered off, dried and recrystallized from the proper solvent to afford the pyridazinone 4. IR spectrum for compound 4 exhibits vNH 3285,vCHAr 3050,vCO $1670 \mathrm{~cm}^{-1}$, the ${ }^{1} \mathrm{H}-\mathrm{NMR}$ spectrum for $4 \mathrm{~b}$ in DMSO $2.2(\mathrm{~s}, 9 \mathrm{H}), 2.8(2 \mathrm{dd}, 2 \mathrm{H}$,diasterotopic protons), 3.4 (dd,1H,CH-CO , pyridazine moiety),6.8-7.4(m,7H,ArH),8.5 and 13.2(bs,2H,NH).

2-Phenyl-4-(2,4-dimethyl or 2,5-dimethyl)phenyl and/or phenyl-phenylsulfanyl5(4H)-furanone (5)

A mixture of propionic acids $2(0.01 \mathrm{~mol})$ and acetic anhydride $(0.01 \mathrm{~mol})$ was heated under reflux for $3 \mathrm{hr}$. The reaction mixture was concentrated. The solid was separated out, filtered off, dried and recrystallized from the proper solvent to afford the furanone 5. IR Spectra for compounds 5 exhibit vCHAr 3050, vCO 1762-1750 $\mathrm{cm}^{-1}$. ${ }^{1} \mathrm{H}-\mathrm{NMR}$ spectrum for $5 \mathrm{~b}$ in (DMSO) 2.5 $\left(\mathrm{s}, 9 \mathrm{H}, \mathrm{CH}_{3}\right) \quad, 4(\mathrm{dd}, 1 \mathrm{H}, \quad \mathrm{CH}-\mathrm{CO}), 6.7(\mathrm{~s}, 1 \mathrm{H}, \quad \mathrm{CH}-\mathrm{R}$, furanone moiety), 7.5$7.9(\mathrm{~m}, 7 \mathrm{H}, \mathrm{ArH}), 13.5(\mathrm{~s}, 1 \mathrm{H}, \mathrm{NH}$, exchangeable proton). EIMS of $5 \mathrm{a}$ and $5 \mathrm{c} \mathrm{m} / \mathrm{z}$ at 322 and 325 corresponding to molecular ion peak, respectively .

3- Acetylamino phenyl-5-[(2,4- and 2,5-dimethyl) phenyl and/or phenyl sulfanyl]-4,5,6-trihydro1,2-oxazin-6-one (6)

A mixture of $2(0.01 \mathrm{~mol})$ and hydroxyl amine hydrochloride $(0.01 \mathrm{~mol})$ was heated under reflux in pyridine $(30 \mathrm{ml})$ for $3 \mathrm{hr}$. The reaction mixture was

Egypt. J. Chem. 54, No. 1 (2011) 
filtered off on hot, then left to cool and pour into ice/ $\mathrm{HCl}$. The solid was separated out, filtered off, dried and recrystallized from the proper solvent to give 1,2 oxazin-6-one 6.IR vNH 3220, vCHAr 3050, vSH 2300, vCO $1735 \mathrm{~cm}^{-1}$. ${ }^{1} \mathrm{H}-\mathrm{NMR}$ of $6 \mathrm{a}$ in DMSO $2.45\left(\mathrm{~s}, 9 \mathrm{H}, \mathrm{CH}_{3}\right), 2.9$ (2dd,2H, diasterotopic protons, $\left.\mathrm{CH}_{2}-\mathrm{CH}\right)$, $3.6(\mathrm{dd}, 1 \mathrm{H}, \mathrm{CH}-\mathrm{CO}$, oxazine moiety) ,7.3-7.9(m,7H), 11.3 (s,1H,exchangeable $\mathrm{NH})$.

2-Phenyl-3-acetylamino phenyl-5-phenyl sulfanyl-1, 4, 5,6-tetrahydro-pyridazin6-one(7)

A mixture of acid $2 c(0.01 \mathrm{~mol})$ and phenyl hydrazine $(0.01 \mathrm{~mol})$ in ethanol $(30 \mathrm{ml})$ was heated under reflux for $3 \mathrm{hr}$. The reaction mixture was poured on ice after cooling. The separated solid was filtered off, dried and recrystallized from ethanol . IR vCHAr 3050 , vCHAli $2886 \mathrm{~cm}^{-1}$, vCO 1687-1670. ${ }^{1} \mathrm{H}-$ NMR in (DMSO) $2.5(\mathrm{~s}, 6 \mathrm{H}), 3.1$ (2dd,2H,disterotopicprotons) 3.7 (dd,1H,CH-) [pyridazine moiety],7.17.9(m,14H), $11.4(\mathrm{~s}, 1 \mathrm{H}, \mathrm{NH})$.

2- Alkyl-4- (2,4-/ 2,5-dimethyl) phenyl-6-Acetylamino phenyl-1, 4, 5,6-tetrahydro pyridazinone ( 8 )

A mixture of pyridazinone $4 \mathrm{a}, \mathrm{b}(0.01 \mathrm{~mol})$ and alkyl halide $(0.01 \mathrm{~mol})$ namely methyl iodide, ethyl iodide, allyl bromide, benzyl chloride ,acetyl chloride and ethyl chloro acetate in dry pyridine was refluxed for $3 \mathrm{hr}$. The reaction mixture was poured on ice/ $\mathrm{HCl}$. The separated solid was filtered off, dried and recrystallized from the proper solvent to afford alkyl pyridazine 8 . IR vCHAr 3070-3050, vCHAil 1945-2886, vCO 1730-1670 $\mathrm{cm}^{-1} .{ }^{1} \mathrm{H}-\mathrm{NMR}$ of 8a in (DMSO) $2.3\left(\mathrm{~s}, 9 \mathrm{H}, \mathrm{CH}_{3}\right), 3.1$ (2dd,2H,disterotopicprotons) , 3.7 (dd, $\left.1 \mathrm{H}, \mathrm{CH}-\right)$ [pyridazine moiety] , 7.17.9 (m,7H,ArH) ${ }^{1} \mathrm{H}-\mathrm{NMR}$ of $8 l$ in (DMSO) 1.1 $\left(\mathrm{t}, 3 \mathrm{H}, \mathrm{CH}_{2} \mathrm{CH}_{3}\right) \quad 2.3\left(\mathrm{~s}, 9 \mathrm{H}, \mathrm{CH}_{3}\right) \quad, 3.1$ (2dd,2H,disterotopic protons) , 3.7 $(\mathrm{dd}, 1 \mathrm{H}, \mathrm{CH}-)$ [pyridazine moiety], $4.1 \quad\left(\mathrm{q}, 2 \mathrm{H} . \mathrm{CH}_{2} \mathrm{CH}_{3}\right), 4.8 \quad\left(\mathrm{~s}, 2 \mathrm{H}, \mathrm{N}-\mathrm{CH}_{2}\right) 7.1-$ 7.9(m,7H,ArH). EIMS for compounds $8_{\mathrm{b}}$ and $8_{\mathrm{g}}$ exhibited $\mathrm{m} / \mathrm{z} 348$ and 426 respectively corresponding to $\mathrm{M}$.The compound $8_{\mathrm{k}}$ exhibited $\mathrm{mlz} 336$ corresponding to $\left(\mathrm{M}-\mathrm{COCH}_{3}\right)$. 
TABLE 1. Characterization and physical data for synthesized compounds .

\begin{tabular}{|c|c|c|c|c|c|c|c|c|}
\hline \multirow{2}{*}{$\begin{array}{c}\text { Cpd. } \\
\text { No }\end{array}$} & \multirow[t]{2}{*}{ M.P. ${ }^{\circ} \mathbf{C}$} & \multirow{2}{*}{$\begin{array}{c}\text { Yield } \\
\%\end{array}$} & \multirow{2}{*}{$\begin{array}{c}\text { Solvent } \\
\text { Of } \\
\text { Cryst. } \\
\end{array}$} & \multirow[t]{2}{*}{ Formula M.Wt } & \multicolumn{4}{|c|}{ Analysis \% calcd/found } \\
\hline & & & & & $\mathrm{C}$ & $\mathrm{H}$ & $\mathrm{N}$ & $\mathrm{S}$ \\
\hline $2 \mathrm{a}$ & $205-207$ & 70 & Ethanol & $\mathrm{C}_{20} \mathrm{H}_{21} \mathrm{NO}_{4}(339)$ & $\begin{array}{l}70.7 \\
70.4\end{array}$ & $\begin{array}{c}6.5 \\
6.25\end{array}$ & $\begin{array}{l}4.1 \\
3.9\end{array}$ & - \\
\hline $2 \mathrm{~b}$ & $218-220$ & 60 & Ethanol & $\mathrm{C}_{20} \mathrm{H}_{21} \mathrm{NO}_{4}(339)$ & $\begin{array}{l}70.7 \\
70.4 \\
\end{array}$ & $\begin{array}{c}6.5 \\
6.25 \\
\end{array}$ & $\begin{array}{l}4.1 \\
3.9 \\
\end{array}$ & - \\
\hline 3 & $210-212$ & 55 & Ethanol & $\mathrm{C}_{18} \mathrm{H}_{17} \mathrm{NO}_{4} \mathrm{~S}(343)$ & $\begin{array}{l}62.9 \\
62.6\end{array}$ & $\begin{array}{l}4.9 \\
4.3\end{array}$ & $\begin{array}{l}4.1 \\
4.3\end{array}$ & $\begin{array}{l}9.3 \\
9.4\end{array}$ \\
\hline $4 \mathrm{a}$ & $150-153$ & 80 & Ethanol & $\mathrm{C}_{20} \mathrm{H}_{21} \mathrm{~N}_{3} \mathrm{O}_{2}(335)$ & $\begin{array}{l}71.6 \\
71.4\end{array}$ & $\begin{array}{l}6.3 \\
6.5\end{array}$ & $\begin{array}{l}12.8 \\
12.7\end{array}$ & - \\
\hline $4 \mathrm{~b}$ & $140-142$ & 75 & Ethanol & $\mathrm{C}_{20} \mathrm{H}_{21} \mathrm{~N}_{3} \mathrm{O}_{2}(335)$ & $\begin{array}{l}71.6 \\
71.4\end{array}$ & $\begin{array}{l}6.3 \\
6.5\end{array}$ & $\begin{array}{l}12.8 \\
12.7\end{array}$ & - \\
\hline $4 \mathrm{c}$ & $105-107$ & 50 & Ethanol & $\mathrm{C}_{18} \mathrm{H}_{17} \mathrm{~N}_{3} \mathrm{O}_{2} \mathrm{~S}(339)$ & $\begin{array}{l}63.7 \\
63.4\end{array}$ & $\begin{array}{l}5.0 \\
5.2\end{array}$ & $\begin{array}{l}12.4 \\
12.7\end{array}$ & $\begin{array}{l}9.4 \\
9.6\end{array}$ \\
\hline $5 \mathrm{a}$ & $123-125$ & 85 & Ethanol & $\mathrm{C}_{20} \mathrm{H}_{19} \mathrm{NO}_{3}(321)$ & $\begin{array}{l}74.7 \\
74.4\end{array}$ & $\begin{array}{l}5.9 \\
5.5\end{array}$ & $\begin{array}{l}4.4 \\
4.6\end{array}$ & - \\
\hline $5 \mathrm{~b}$ & $130-133$ & 70 & Ethanol & $\mathrm{C}_{20} \mathrm{H}_{19} \mathrm{NO}_{3}(321)$ & $\begin{array}{l}74.7 \\
74.6\end{array}$ & $\begin{array}{l}5.9 \\
5.6\end{array}$ & $\begin{array}{l}4.4 \\
4.4\end{array}$ & - \\
\hline $55_{c}$ & $125-128$ & 60 & Ethanol & $\mathrm{C}_{18} \mathrm{H}_{14} \mathrm{NO} 3 \mathrm{~S}(324)$ & $\begin{array}{l}66.6 \\
66.3\end{array}$ & $\begin{array}{l}4.3 \\
4.3\end{array}$ & $\begin{array}{l}4.3 \\
4.1\end{array}$ & $\begin{array}{l}9.8 \\
9.8\end{array}$ \\
\hline $6 a$ & $220-223$ & 65 & Butanol & $\mathrm{C}_{20} \mathrm{H}_{20} \mathrm{~N}_{2} \mathrm{O}_{3}(336)$ & $\begin{array}{l}71.4 \\
71.7\end{array}$ & $\begin{array}{l}5.9 \\
5.7\end{array}$ & $\begin{array}{l}8.3 \\
8.6\end{array}$ & - \\
\hline $6 b$ & $225-228$ & 60 & Butanol & $\mathrm{C}_{20} \mathrm{H}_{20} \mathrm{~N}_{2} \mathrm{O}_{3}(336)$ & $\begin{array}{l}71.4 \\
71.2\end{array}$ & $\begin{array}{l}5.9 \\
5.6\end{array}$ & $\begin{array}{l}8.3 \\
8.3\end{array}$ & - \\
\hline $6 c$ & $200-204$ & 50 & Dioxan & $\mathrm{C}_{18} \mathrm{H}_{16} \mathrm{~N}_{2} \mathrm{O}_{3} \mathrm{~S}(340)$ & $\begin{array}{l}63.5 \\
63.4\end{array}$ & $\begin{array}{l}4.7 \\
4.3 \\
\end{array}$ & $\begin{array}{l}8.3 \\
8.6\end{array}$ & $\begin{array}{l}9.4 \\
9.8\end{array}$ \\
\hline $7 \mathrm{c}$ & $110-113$ & 50 & Ethanol & $\mathrm{C}_{24} \mathrm{H}_{21} \mathrm{~N}_{3} \mathrm{O}_{2} \mathrm{~S}(415)$ & $\begin{array}{l}69.4 \\
69.1\end{array}$ & $\begin{array}{l}5.1 \\
5.4\end{array}$ & $\begin{array}{l}10.1 \\
10.4\end{array}$ & $\begin{array}{l}7.7 \\
7.1\end{array}$ \\
\hline $8 \mathrm{a}$ & $128-132$ & 70 & Ethanol & $\mathrm{C}_{21} \mathrm{H}_{23} \mathrm{~N}_{3} \mathrm{O}_{2}(349)$ & $\begin{array}{l}72.2 \\
72.4 \\
\end{array}$ & $\begin{array}{l}6.6 \\
6.7 \\
\end{array}$ & $\begin{array}{l}12.0 \\
11.9 \\
\end{array}$ & - \\
\hline $8 \mathrm{~b}$ & $133-335$ & 80 & Ethanol & $\mathrm{C}_{21} \mathrm{H}_{23} \mathrm{~N}_{3} \mathrm{O}_{2}(349)$ & $\begin{array}{l}72.2 \\
72.0\end{array}$ & $\begin{array}{l}6.6 \\
6.4\end{array}$ & $\begin{array}{l}12.0 \\
11.9\end{array}$ & - \\
\hline $8 \mathrm{c}$ & $155-158$ & 50 & Ethanol & $\mathrm{C}_{22} \mathrm{H}_{25} \mathrm{~N}_{3} \mathrm{O}_{2}(363)$ & $\begin{array}{l}72.7 \\
72.1\end{array}$ & $\begin{array}{l}6.9 \\
6.4\end{array}$ & $\begin{array}{l}11.6 \\
11.3\end{array}$ & - \\
\hline $8 \mathrm{~d}$ & $162-165$ & 50 & Ethanol & $\mathrm{C}_{22} \mathrm{H}_{25} \mathrm{~N}_{3} \mathrm{O}_{2}(363)$ & $\begin{array}{l}72.7 \\
72.1\end{array}$ & $\begin{array}{l}6.9 \\
6.4\end{array}$ & $\begin{array}{l}11.6 \\
11.3\end{array}$ & - \\
\hline $8 \mathrm{e}$ & $175-178$ & 70 & Ethanol & $\mathrm{C}_{23} \mathrm{H}_{25} \mathrm{~N}_{3} \mathrm{O}_{2}(375)$ & $\begin{array}{l}73.6 \\
73.7\end{array}$ & $\begin{array}{l}6.6 \\
6.6\end{array}$ & $\begin{array}{l}11.2 \\
11.5\end{array}$ & - \\
\hline $8 f$ & $180-183$ & 60 & Ethanol & $\mathrm{C}_{23} \mathrm{H}_{25} \mathrm{~N}_{3} \mathrm{O}_{2}(375)$ & $\begin{array}{l}73.6 \\
73.3\end{array}$ & $\begin{array}{l}6.6 \\
6.4\end{array}$ & $\begin{array}{l}11.2 \\
11.0\end{array}$ & - \\
\hline $8 g$ & $205-208$ & 55 & Dioxan & $\mathrm{C}_{27} \mathrm{H}_{27} \mathrm{~N}_{3} \mathrm{O}_{2}(425)$ & $\begin{array}{l}76.2 \\
76.4\end{array}$ & $\begin{array}{l}6.4 \\
6.5\end{array}$ & $\begin{array}{l}9.9 \\
9.4\end{array}$ & - \\
\hline $8 \mathrm{~h}$ & $200-203$ & 60 & Dioxan & $\mathrm{C}_{27} \mathrm{H}_{27} \mathrm{~N}_{3} \mathrm{O}_{2}(425)$ & $\begin{array}{l}76.2 \\
76.0 \\
\end{array}$ & $\begin{array}{l}6.4 \\
6.5 \\
\end{array}$ & $\begin{array}{l}9.9 \\
9.6 \\
\end{array}$ & - \\
\hline $8 \mathrm{i}$ & $180-183$ & 75 & Dioxan & $\mathrm{C}_{22} \mathrm{H}_{25} \mathrm{~N}_{3} \mathrm{O} 3(377)$ & $\begin{array}{l}70.0 \\
70.5 \\
\end{array}$ & $\begin{array}{l}6.1 \\
6.3 \\
\end{array}$ & $\begin{array}{l}11.1 \\
11.4 \\
\end{array}$ & - \\
\hline $8 \mathrm{j}$ & $185-188$ & 80 & Dioxan & $\mathrm{C}_{22} \mathrm{H}_{25} \mathrm{~N}_{3} \mathrm{O} 3(377)$ & $\begin{array}{l}70.0 \\
70.0\end{array}$ & $\begin{array}{l}6.1 \\
6.2\end{array}$ & $\begin{array}{l}11.1 \\
11.0\end{array}$ & - \\
\hline $8 \mathrm{k}$ & $105-108$ & 85 & Ethanol & $\mathrm{C}_{24} \mathrm{H}_{27} \mathrm{~N}_{3} \mathrm{O}_{4}(421)$ & $\begin{array}{l}68.4 \\
68.5\end{array}$ & $\begin{array}{l}6.4 \\
6.3\end{array}$ & $\begin{array}{l}10.0 \\
10.0\end{array}$ & - \\
\hline $88_{1}$ & $115-118$ & 80 & Ethanol & $\mathrm{C}_{24} \mathrm{H}_{27} \mathrm{~N}_{3} \mathrm{O}_{4}(421)$ & $\begin{array}{l}68.4 \\
68.4\end{array}$ & $\begin{array}{l}6.4 \\
6.2\end{array}$ & $\begin{array}{l}10.0 \\
10.3\end{array}$ & - \\
\hline
\end{tabular}

Egypt. J. Chem. 54, No. 1 (2011) 


\section{References}

1. Coelho, A., Sotelo, E. and Ravina, E., Pyridazine derivatives. Part 33: q Sonogashira approaches in the synthesis of 5-substituted-6-phenyl-3 (2H)- pyridazinones. Tetrahedron, 59 , 2477-2484, (2003)

2. Demirayak, S., Karaburun, A.C. and Beis, R., Some pyrrole substituted aryl pyridazinone and phthalazinone derivatives and their antihypertensive activities. Eur. J. Med. Chem. 39, 1089-1095 (2004)

3. Siddiqui, A.A. and Wani, S.M., Ind. J. Chem. 43B, 1574-1579 (2004)

4. Monge, A., Parrado, P., Font, M. and Alvarez, E.F., J. Med. Chem. 30 (6), 10291035 (1987)

5. Rubat, C., Coudert, P., Refouvelet, B., Tronche, P. and Bastide, P., Chem. Pharm. Bull. 38 (11), 3009-3013 (1990)

6. Sircar, I., Weishaar, R.E., Kobylarz, D., Moos, W.H. and Bristol, J.A., J. Med. Chem. 30, 1955-1962 (1987)

7. Longo, J.G., Verde, I. and Castro, M.E., J. Pharm. Sci. 82, 286-290 (1993)

8. Akahane, A., Katayama, H. and Mitsunaga, T., J. Med. Chem. 42, 779-783 (1999)

9. Livermone, D.G.H., Bethell, R.C. and Cammack, N., J. Med. Chem. 36, 3784-3794 (1993)

10. a) Malinka, W., Redzicka, A. and Lozach, O., Il Farmaco. 59, 457-462 (2004) b) Malinka, W., Redzika, A., Jastrzebska, M., Wiesek, Filipek, B., Dybala, M., Karczmarzyk, Z., Urbanczyk-Lipkowska, Z. and Kalicki. P., Derivatives of pyrrolo [3,4-d] pyrida- zinone, a new class of analgesic agent. Eur. J. Med. Chem. 64 (10), 4992-9 (2011)

11. Abouzid, K., Hakeem, M.A., Khalil, O. and Maklad, Y., Bioorg. Med. Chem. 16, 382-389 (2008)

12. Combs, D.W., Rampulla, M.S., Bell, S.C., Klaubert, D.H., Tobia, A.J., Falotico, R., Haertlein, B., Weiss, C.L. and Moore, J.B., J. Med. Chem. 22, 380-386 (1990)

13. Robertson, D.W., Jones, N.D., Krushinski, J.H., Pollock, G.D., Swartzendruber, J.K. and Scott Hayes, J., J. Med. Chem. 30, 623-627 (1987)

14. Archan, S. and Toller, W., Curr. Opin. Anesthesiol, 21 (1) 78-84 (2008)

15. Sotelo, E., Coelho, A. and Ravina, E., Tetrahedron Lett. 44, 4459-4462 (2003)

16. Siddiqui, A.A., Ahmad, S.R. and Hussain, S.A., Acta Pol. Pharm. 64 (2), 223-228 (2008) 
17. Siddiqui, A.A., Ashok, and Wani, S.M., Ind. J. Heterocycl. Chem. 13, 257-260 (2004)

18. Bansal, R., Kumar, D., Carron, R. and de la Calle, C., Eur. J. Medicinal Chem. 44, 4441-4447 (2009)

19. Siddiqui, A.A., Mishra, R. and Shaharyar, M., Eur. J. Medicinal Chem. 45, 2283-2290 (2010)

20. Papa, D., Schwenk, E., Villain, F. and Klingsberg, E., Am.J.Chem. 70, 3356 (1948)

21. Pumerer and Buchta, Ber. 69, 1005 (1936)

22. Sammour, A. and El-Hashash, M., J. Prakt. Chemie, 314, 906 (1972)

23. El-Hashash, M., El-Kady, M. and Mohamed, M., Reaction of 2-aryl-3-(4bromobenzoyl) propionic acid via Friedel Crafts alkylation of aromatic hydrocarbons with 3-(4-bromo-) benzoyl acrylic acid. Indian. J. Chem. 18B, 136 (1979).

24. El-Hashash, M., El-Kady, M. and Mohamed, M., Indian.J.Chem. 19B (1980)

25. El-Hashash, M., El-Kady, M. and Hosni, G., Roumaine.J.Chem. 24, 839 (1979)

26. Mohamed, M., El-Hashash, M. and El-Kady, M., Roumaine.J.Chem. 24,1381 (1979)

27. a) El-Hashash, M.A., Mohamed, M.M., Islam, I. and Abo-Baker, O.A., Behavior of 3-(4-chloro-3-methylbenzoyl) acrylic acid towards carbon nucleophiles under Micheal reaction Conditions, Indian. J.Chem. 21B,735 (1982). b) Mohamed M.M., El-Hashash, M.A., Islam, I. and Abo-Baker, O.A., J. Revue Roumaine de Chimie, 27865 (1982)

28. Salim, M., El-Hashash, M., Harb, N. and Marzouk, M., Pak.J.Chem. 479 (1986)

29. Rizk, S.A., El-Hashash, M.A. and Mostafa, K.K., Utility of $\beta$-aroyl acrylic acid in heterocyclic synthesis. Egypt, J. Chem. 51 (5), 611- 621 (2008)

30. Pant, U., Preti, U., Pant, S., Dandia, A. and Patnaik, G., Phos. Sul. J.Chem. 126, 193-199 (1997)

31. Mahmoud, M., Soliman, E., Ibrahim, G. and Rabie, M., Phos. Sul. J.Chem. 139, 97-106 (1998)

32. Zou, X., Jin, G. and Zhang, Z., J. Food Agri. Chem. 50(6), 451-1454 (2002)

33. Lee, S., Kweon, D., Kang, Y., Kim, K., Cho, S. and Yoon, Y., J.Curr. Org. Chem. 8, 1463-1480 (2004)

34. Orru, R. and De-Greaf, M., Synthesis. J. Chem. 10, 1471-1499 (2003)

35. IUgi, A., Domling, and Werner, B., J.Hetero.Chem. 37,647-658 (2000)

Egypt. J. Chem. 54, No. 1 (2011) 
36. Mark, S., John, C., Todd, A., Jennifer, M., Matthew, J., Adam, G., Biswanath, D., Sutian, E., Lily, C., Jeff, R., Slere, B., Eric, S. and Michael, J., J. Bioorg.Med.Chem. 16, 4257-4261 (2006)

37. Sukuroglu, M., Caliskan Ergun, B., Unlu, S., Sahin, M.F., Kupel, E., Yesilada, E. and Banoglu, E., Synthesis, analgesic and anti-Inflammatory activities of [6-(3,4dimethyl-4-chloropyrazol-1-yl)-3(2H)-pyridazinon-2-yl] acetamide. Arch. Pharm. Res. 28 (5), 509 (2005)

38. Dogrver, S., Sahin, M., Unlv, S. and Shigervs I., Arch.Pharm. 333, 79 (2000)

39. Breda, S., Reva, I. and Fausto, R., Vibrational Spectroscopy, 50, 57-67 (2009)

40. Ogliaruso, M. and Wolle, J., Synthesis of Lactone and Lactam. Wiley. New York (1993)

41. Moos, W., Humblet, C., Sircar, I., Thner, C., Weishar, R. and Bristol, J., J. Med. Chem. 30,1972 (1987)

42. Coates, W., Comprehensive Heterocyclic Chem. (Katritzky), Pyridazine and their Benzoderivatives (1-1183) (1996)

(Received 4/1/2011;

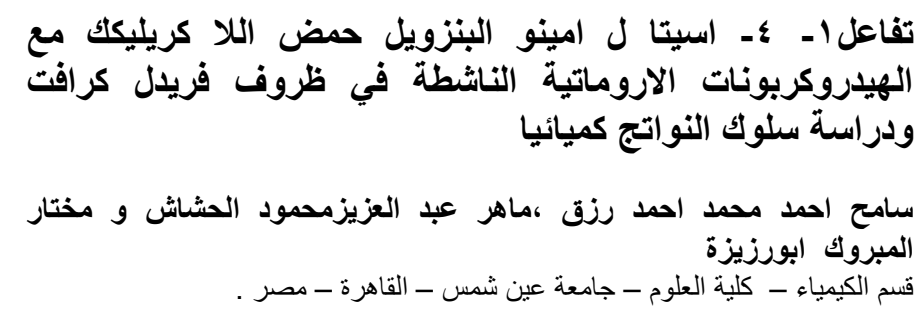

1- تحضير بعض الاحماض البروبيونيك الحاملة مجموعات الاريل و الكبرينو

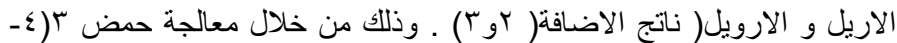

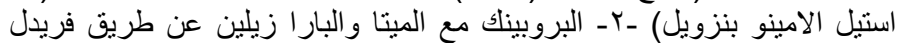

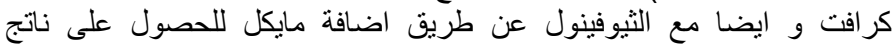

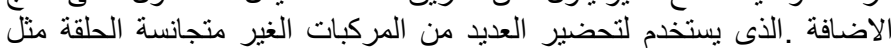

البيريدازينون و الاكز ازينون و الفيور انون.

ب-اجر اء بعض التجارب على مشتقات البيريدازينون للحصول على مركبات اكثر

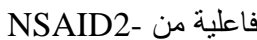

ب-اثبات المركبات المحضرة بأجهزة التحاليل الدقيقة مثل الاشعة تحت الحمراء

و الرنين المغناطيسى والكتلة الاكترونى. بأنى الكرة.

Egypt. J. Chem. 54, No. 1 (2011) 